\title{
A Trial of Complement Inhibition in a Patient with Cryoglobulin-Induced Glomerulonephritis
}

\author{
Patricia Hirt-Minkowski ${ }^{a} \quad$ Marten Trendelenburg ${ }^{b, c}$ \\ Isabel Gröschl ${ }^{a}$ Andreas Fischer ${ }^{\mathrm{e}}$ Ingmar Heijnen ${ }^{d}$ \\ Jürg A. Schifferli ${ }^{\text {b }}$ \\ ${ }^{a}$ Clinic for Transplantation Immunology and Nephrology, ${ }^{b}$ Division of Internal \\ Medicine, and ${ }^{\mathrm{C} C l i n i c a l}$ Immunology, University Hospital Basel, and ${ }^{\mathrm{d}}$ Medical \\ Immunology, Laboratory Medicine, University Hospital Basel, Basel, and \\ ${ }^{\text {e}}$ Division of Nephrology, Kantonsspital Lucerne, Lucerne, Switzerland
}

\section{Key Words}

Cryoglobulinemia $\cdot$ Glomerulonephritis $\cdot$ Complement inhibition

\begin{abstract}
Cryoglobulinemia induces an immune complex-mediated glomerulonephritis that is characterized by the presence of large immune deposits, including complement $\mathrm{C} 3$ and $\mathrm{C5}$ b9 , marked macrophage influx and mesangial cell proliferation. The precise role of complement in cryoglobulin-induced glomerulonephritis in humans remains unclear, whereas in mice there has been evidence that complement activation might be a central factor favoring glomerular inflammation, particularly by the recruitment of neutrophils. We report on an exceptional case of cryoglobulin-induced glomerulonephritis in a patient with mixed essential cryoglobulinemia type II. The clinical features included relapsing proteinuria and renal function impairment that were controlled by plasmapheresis. Complement was low in plasma and two renal biopsies at 1-year interval showed prominent immunoglobulin and complement deposits, with unusual high numbers of neutrophils. In a 1-patient clinical trial, we tested whether the monoclonal anti-C5 antibody eculizumab would be sufficient to control renal function at the time of a relapse. Although during the initial weeks renal function was stabilized, slow increase in creatinine could not be controlled by this treatment, so that plasmapheresis was reinstituted. This result suggests that despite evidence for a role of complement in enhancing renal damage in this patient, other inflammatory processes dominated.
\end{abstract}




\section{Introduction}

In humans, cryoglobulinemia can be responsible for a membranoproliferative glomerulonephritis (MPGN), which is characterized by the formation and/or deposition of immunoglobulins (Igs) and complement in the glomeruli, followed by an influx of inflammatory cells [1-3]. However, the mechanisms inducing the glomerular inflammation are not fully understood. Complement is well known to mediate the processing of immune complexes [4]. Since the early studies to investigate complement activation by immune complexes in experimental nephrotoxic nephritis, the complement system has been considered to play an important proinflammatory role in the glomerulus $[5,6]$. In cryoglobulinemia, however, there is no clear evidence that complement activation is central in mediating tissue damage by immune deposits. Results have been obtained in mice by Trendelenburg et al. [7], who analyzed the role of complement in the pathogenesis of initial glomerular inflammation induced by murine 6-19 IgG3 monoclonal cryoglobulins. They used two different strains of mice developing either type I or II cryoglobulinemia. Their results indicated that complement $\mathrm{C} 5$ has a predominant role in neutrophil recruitment as C5-deficient mice had significantly reduced glomerular infiltration by polymorphonuclear neutrophils [7]. This is of particular interest, as the monoclonal complement C5-blocking antibody eculizumab could offer a promising therapeutic option for patients with cryoglobulininduced glomerulonephritis.

Cryoglobulinemia is a rare disease and is characterized by Igs that precipitate in the cold. Depending on the clonality of the precipitating Igs, three types of cryoglobulinemia can be distinguished [8]. Type I cryoglobulinemia consists of a single monoclonal cryoprecipitable Ig, whereas type II and III cryoglobulins are mixed, i.e. composed of monoclonal (type II) and polyclonal (type III) antibodies with rheumatoid factor activity against other Igs. Mixed cryoglobulinemia may be associated with numerous infectious and immunological diseases as well as lymphatic malignancies. In particular, mixed cryoglobulinemia type II is strongly associated with chronic hepatitis $\mathrm{C}$, and nowadays rarely appears as an isolated disease, i.e. the so-called essential cryoglobulinemia [9]. Clinically, cryoglobulinemia manifests itself with symptoms like weakness and arthralgias and variable cutaneous and visceral organ involvement [9, 10]. Overall, the prognosis is dependent on the underlying disease and affected organs. It is poorer in patients with renal involvement, liver failure, lymphoproliferative disease and malignancies [9]. The treatment of cryoglobulinemia is challenging. Steroids suppress inflammation with success in some patients, but additional plasmapheresis to remove circulating cryoglobulins and immunosuppressive treatment to inhibit the formation of new cryoglobulins are often necessary [11-13]. All of these therapies are characterized by serious side effects.

Eculizumab (Soliris; Alexion Pharmaceuticals, Cheshire, Conn., USA) is a monoclonal antibody that targets complement $\mathrm{C} 5$ and prevents the cytolytic and proinflammatory effects of complement activation [14]. Eculizumab is approved for the treatment of patients with paroxysmal nocturnal hemoglobinuria. It effectively reduces intravascular hemolysis, thereby improving anemia and quality of life and decreases transfusion requirements $[15,16]$. Since the initial validation of inhibition of a complement component as a therapeutic target, the field for the use of eculizumab has been expanded [17]. Recently, eculizumab has been approved from the FDA for the 
treatment of patients with atypical hemolytic uremic syndrome, in whom the control of complement activation is insufficient. Furthermore, eculizumab has been successfully used preemptive prior to kidney transplantation $[18,19]$ or postoperatively to prevent disease recurrence in the allograft [20-22] in several cases with complement disorderassociated atypical hemolytic uremic syndrome.

Here, we report on an exceptional case of mixed essential cryoglobulinemia with severe glomerulonephritis characterized by a prominent infiltration of the glomeruli by neutrophils. A 1-patient clinical trial of eculizumab was attempted to investigate whether complement inhibition would be sufficient to inhibit this relapsing glomerulonephritis. The initial partial response was, however, not sustained, and the patient returned to intermittent plasmapheresis.

\section{Case Report and Therapeutical Trial}

A 55-year-old woman was diagnosed with a mixed type II cryoglobulinemia (monoclonal IgMkappa and polyclonal IgG) and immune complex-mediated glomerulonephritis in May 2006. She developed an acute nephritic syndrome with pathological urinary sediment (i.e. dysmorphic erythrocytes, cellular casts and leucocytes), high proteinuria (7 g/day), arterial hypertension, volume overload and moderate renal function impairment, which got worse over time. Further, she suffered from weakness and arthralgias, but other organ manifestations besides the kidney were not present. The first renal biopsy showed endocapillary hypercellularity with infiltration of mononuclear cells, an unusual high number of neutrophils, and subendothelial immune deposits, which were positive for IgM as well as complement C3 and C5b-9, resembling acute poststreptococcal glomerulonephritis; however, classical humps were missing (fig. 1). Comprehensive blood examinations revealed cryoglobulins $(0.6 \mathrm{~g} / \mathrm{l})$, a monoclonal IgM $(2 \mathrm{~g} / \mathrm{l})$, hypogammaglobulinemia $(<5 \mathrm{~g} / \mathrm{l})$, and low complement $\mathrm{C} 3$ and $\mathrm{C} 4$ levels. Extensive screening for an infectious disease (i.e. serological testing and PCR for HIV, HCV, HBV, CMV and EBV) and malignancy (bone marrow biopsy, computed tomography of the chest and abdominal organs) was negative. Further, autoantibodies were negative (ANA, ANCA, anti-DNA, antiphospholipid antibodies) except for rheumatoid factor, with a titer $>1 / 10,000$. A second renal biopsy 1 year later showed early stage MPGN with a still high number of neutrophils, which was interpreted as a progression of disease.

The patient was unresponsive to high-dose steroids and subsequently received cyclophosphamide pulse therapy, mycophenolat mofetil and two courses of the monoclonal anti-CD20 antibody rituximab. These immunosuppressive therapies all failed to get the cryoglobulin-induced glomerulonephritis under control. On October 2006, once to twice weekly plasmapheresis was started with success. However, plasmapheresis was complicated by two catheter-related blood stream infections. In addition, she suffered from headache and complained about unpleasant diaphoresis during the procedures. At the request of the patient, plasmapheresis was interrupted several times with prompt disease relapses manifested by an increase in serum creatinine and proteinuria, and reinstitution of intensive plasmapheresis. Thus, we designed a protocol to test whether eculizumab could replace plasmapheresis at the time of a relapse. The protocol was approved by the local ethical committee, and informed consent was provided in accordance with the Declaration of Helsinki. Criteria for treatment success were: (i) prevention of progression as determined by maintenance or improvement in renal function, (ii) reduction of proteinuria, and (iii) no appearance of new extrarenal disease manifestations.

In June 2011, the study was started after the last series of plasma exchange, with a continuous surveillance of renal function and clinical signs of relapse. As expected from previous intervals between plasmapheresis series, it took 3 weeks until relapse. Eculizumab was dosed at $900 \mathrm{mg}$ intravenously every 7 days for 4 weeks, at 1,200 mg 7 days later, and another dose of 1,200 mg 14 days later. At that time of initiating eculizumab therapy, serum creatinine level was $340 \mu \mathrm{mol} / \mathrm{l}$ (eGFR $13 \mathrm{ml} / \mathrm{min}$ ), proteinuria was $8 \mathrm{~g} /$ day and the patient still had nephritic urinary sediment. The patient had been vaccinated against Neisseria meningitis 14 days prior to receive the first dose of eculizumab. Fig. 2 shows the clinical course and renal function (i.e. serum creatinine and proteinuria), before and 
after initiating eculizumab. Blood samples were drawn to control blood concentrations of eculizumab and blockage of the complement system during the treatment. Complement activity was shown to be blocked using a hemolytic assay (CH50) and by the drop to background of the high levels of soluble C5b-9 (MicroVue ${ }^{\circledast}$ Complement SC5b-9 Plus; Quidel Corporation, San Diego, Calif., USA) (data not shown). As expected, complement C3 and C4 levels remained low during eculizumab therapy. The eculizumab infusions were well tolerated and the patient reported an improvement in her well-being. Nevertheless, without plasmapheresis the blockage of the terminal complement was not sufficient to control the disease. Proteinuria remained at high levels of 6-8 g/day, hematuria persisted and renal function remained stable for some weeks before deteriorating. After 7 weeks of therapy, the patient was urgently admitted to hospital because of fluid overload. Plasmapheresis was restarted and renal function returned almost to baseline level (fig. 2).

\section{Discussion}

To our knowledge, this is the first report of a clinical trial to investigate the monoclonal complement inhibitor eculizumab in a patient with mixed cryoglobulinemia and severe glomerulonephritis. The unique features of the cryoglobulinemic MPGN in this patient were the resistance to treatments such as steroids, cyclophosphamide and rituximab, the presence of high numbers of neutrophils in two renal biopsies taken at 1-year interval (on both occasions at the time of a severe relapse), and the prompt response to plasmapheresis. All these features suggested that complement was a major player in the disease process: indeed complement was activated and deposited in the glomeruli, and complement factor C5a is known to be a highly active chemotactic agent for neutrophils. In addition, recently macrophages have been shown to be central to the nephritis in a model of murine cryoglobulinemic MPGN [3].

The improvement felt by the patient and the stabilization of the creatinine level after initiation of eculizumab suggested that complement might be involved in the inflammation and tissue damage induced by cryoglobulins. However, it became evident after 7 weeks that there was no improvement, rather a slow deterioration of renal function with ongoing signs of active nephritis in the urinary sediment. Thus, eculizumab therapy was stopped. Evidently complement blockade at the C5 level alone was insufficient. This observation does, however, not exclude a role for complement in cryoglobulin-induced glomerulonephritis. For instance, we do not know whether complement blockade would prevent a relapse, i.e. whether complement might induce a cascade of inflammatory events, which then become independent of continuous complement activation. Further, one can argue that the blockage of $\mathrm{C} 5$ by eculizumab plays a central role to prevent the chemotactic function of $\mathrm{C} 5 \mathrm{a}$ for the recruitment of neutrophils. Once they have invaded the glomerulus where the inflammatory processes take place, they act independently of complement $\mathrm{C} 5 \mathrm{a}$, which has no proinflammatory effects. This would be an argument for a prophylactic and sustained use of eculizumab in patients with cryoglobulinemia when the disease is in remission and against its use as a salvage therapy in disease relapse, to induce a more prolonged remission or reduce the frequency of relapses. This argumentation is supported by the mouse model reported by Trendelenburg et al. [7], where the C5-deficient mice had impressively less influx of neutrophils, as their experimental model of cryoglobulin-induced glomerulonephritis rather resembles a model of preemptive blockage of C5a to prevent glomerular inflammation just at the beginning and not when the inflammatory process is fully developed as it is in case of a relapse. In addition, eculizumab will not prevent 
the deposition of $\mathrm{C} 3$ fragments and release of C3a, which by themselves might interact with complement receptors on mesangial cells, macrophages/monocytes and neutrophils and activate these cells.

Usually, the development of clinical therapy protocols relies on evidence-based guidelines that mainly focus on randomized, controlled trials; however, for rare diseases, such as in the present case of essential cyoglobulinemia, treatment options rely on similarities to other diseases and the understanding of the pathological process on experimental data obtained in animal models. Since human data of cryoglobulinemia are scarce, the present 1-patient trial report demonstrating the response to eculizumab - whether considered partially positive or negative - might be of use for the medical community.

\section{Acknowledgements}

We thank Repasz Craig, Gene Griffin and Julian Platon from Alexion Pharmaceuticals for the helpful technical assistance with the blood PK/PD studies and for the excellent collaboration. Further, we thank Helmut Hopfer, Clinic for Pathology, University Hospital of Basel, Switzerland, for the delivery of the histological images.

\section{Disclosure Statement}

P.H.M. has received a research grant for the study medication from Alexion Pharmaceuticals. The remaining authors declare no competing financial interests. 

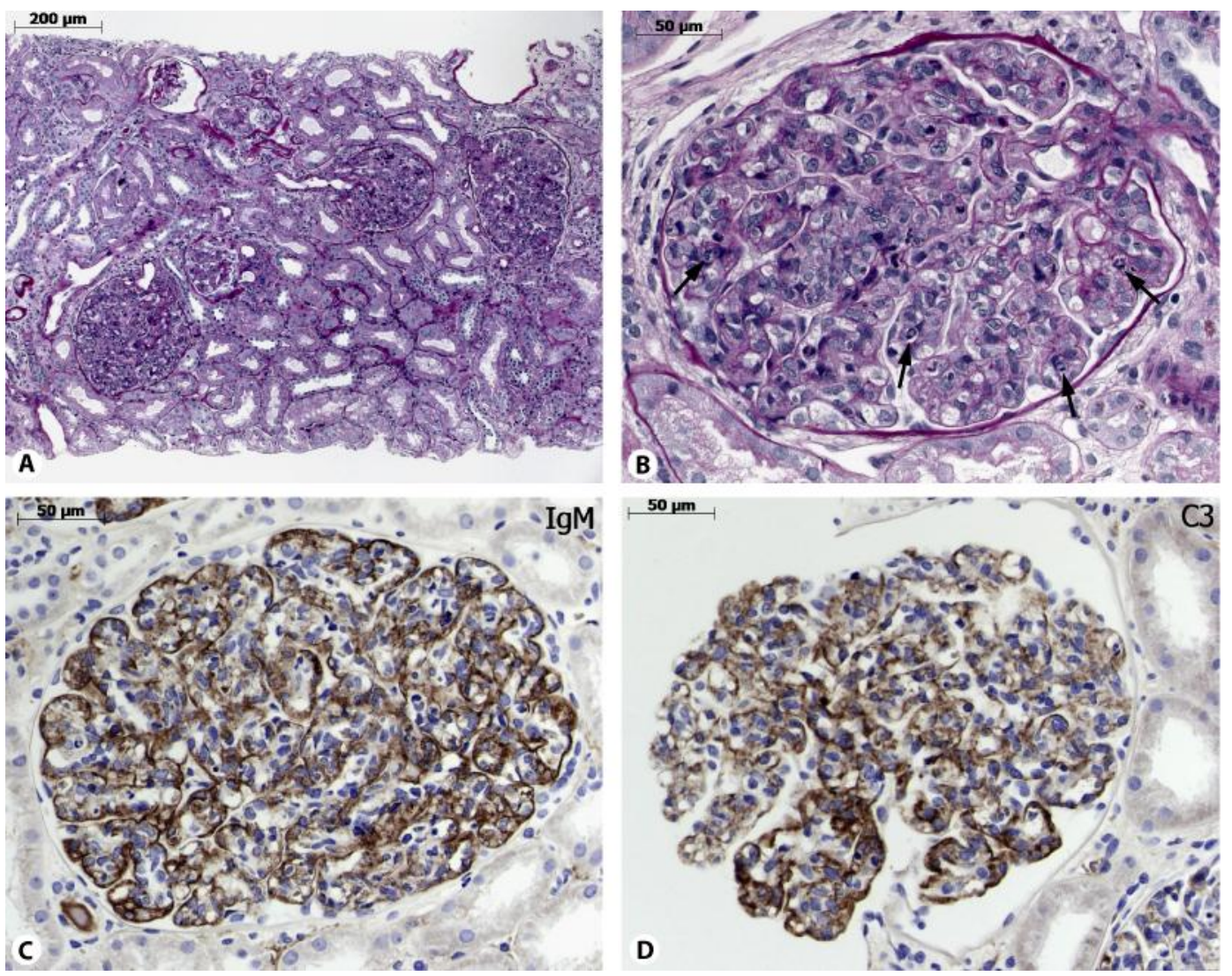

Fig. 1. Renal biopsy findings in a patient with a cryoglobulin-induced immune complex glomerulonephritis. A Overview showing endocapillary glomerulonephritis with impressive hypercellularity. B Section of a glomerulus with mononuclear cells and polymorphonuclear neutrophils (indicated by arrows) in the glomerular convolutes. Immune complex-mediated glomerulonephritis positive for IgM (C) and complement C3 (D) by immunofluorescence staining. 


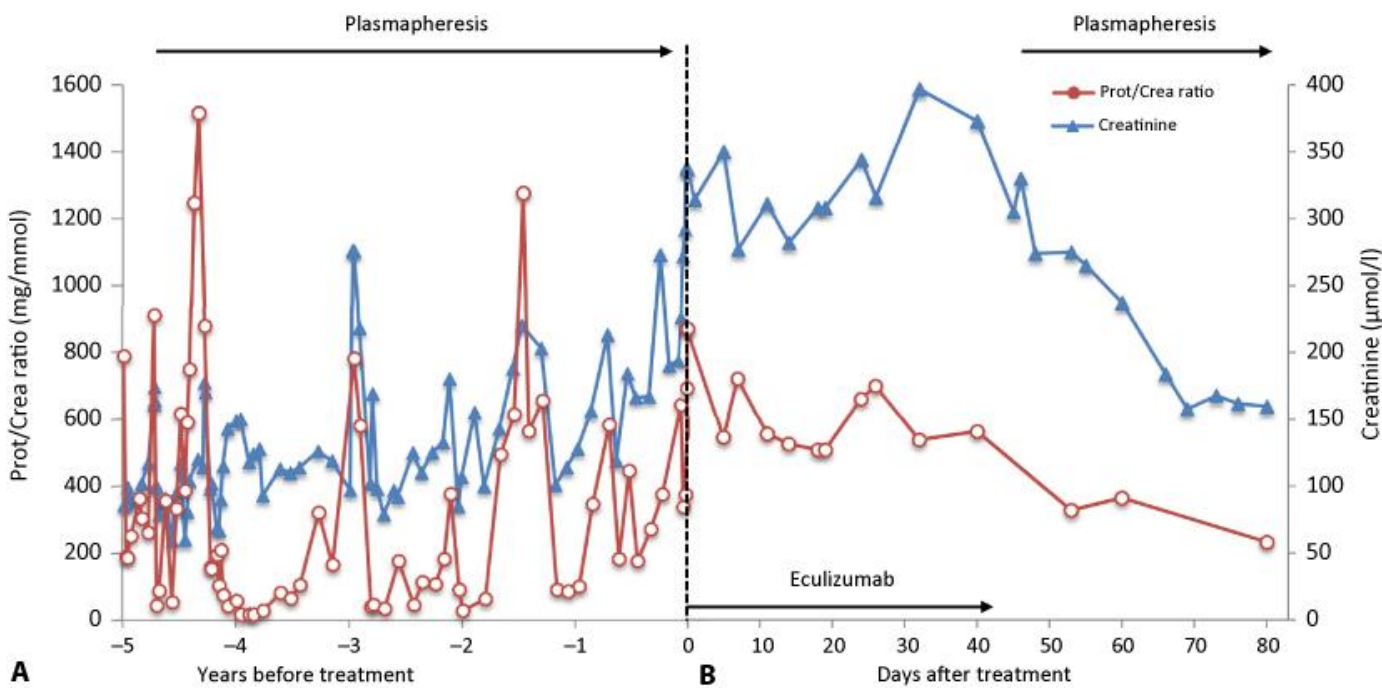

Fig. 2. Clinical course before (A) and after initiation (B) of eculizumab in a patient with a cryoglobulininduced glomerulonephritis. Serum creatinine levels and proteinuria in the years before and after initiation of eculizumab treatment are shown. Plasmapheresis is also indicated. Prot/Crea = Urine protein/creatinine ratio.

\section{References}

1 Garella S: Immunopathologic mechanism of glomerular injury - an overview of current concepts. J Clin Apher 1985;2:387-395.

-2 Adler S, Couser W: Immunologic mechanisms of renal disease. Am J Med Sci 1985;289:55-60.

- 3 Guo S, Wietecha TA, Hudkins KL, Kida Y, Spencer MW, Pichaiwong W, Kojima I, Duffield JS, Alpers CE: Macrophages are essential contributors to kidney injury in murine cryoglobulinemic membranoproliferative glomerulonephritis. Kidney Int 2011;80:946-958.

-4 Schifferli JA, Taylor RP: Physiological and pathological aspects of circulating immune complexes. Kidney Int 1989;35:993-1003.

5 Unanue E, Dixon FJ: Experimental glomerulonephritis. IV. Participation of complement in nephrotoxic nephritis. J Exp Med 1964;119:965-982.

6 Cochrane CG, Unanue E, Dixon FJ: A role of polymorphonuclear leukocytes and complement in nephrotoxic nephritis. J Exp Med 1965;122:99-116.

-7 Trendelenburg M, Fossati-Jimack L, Cortes-Hernandez J, Turnberg D, Lewis M, Izui S, Cook HT, Botto M: The role of complement in cryoglobulin-induced immune complex glomerulonephritis. J Immunol 2005;175:6909-6914.

8 Brouet JC, Clauvel JP, Danon F, Klein M, Seligmann M: Biologic and clinical significance of cryoglobulins. A report of 86 cases. Am J Med 1974;57:775-788.

19 Ferri C: Mixed cryoglobulinemia. Orphanet J Rare Dis 2008;3:25.

10 Lamprecht P, Gause A, Gross WL: Cryoglobulinemic vasculitis. Arthritis Rheum 1999;42:2507-2516.

11 Madore F, Lazarus JM, Brady HR: Therapeutic plasma exchange in renal diseases. J Am Soc Nephrol 1996;7:367-386.

12 Zaja F, De VS, Mazzaro C, Sacco S, Damiani D, De MG, Michelutti A, Baccarani M, Fanin R, Ferraccioli G: Efficacy and safety of rituximab in type II mixed cryoglobulinemia. Blood 2003;101:3827-3834.

13 Ferri C, Moriconi L, Gremignai G, Migliorini P, Paleologo G, Fosella PV, Bombardieri S: Treatment of the renal involvement in mixed cryoglobulinemia with prolonged plasma exchange. Nephron 1986;43:246253. 
14 Thomas TC, Rollins SA, Rother RP, Giannoni MA, Hartman SL, Elliott EA, Nye SH, Matis LA, Squinto SP, Evans MJ: Inhibition of complement activity by humanized anti-C5 antibody and single-chain Fv. Mol Immunol 1996;33:1389-1401.

15 Hillmen P, Hall C, Marsh JC, Elebute M, Bombara MP, Petro BE, Cullen MJ, Richards SJ, Rollins SA, Mojcik $\mathrm{CF}$, Rother RP: Effect of eculizumab on hemolysis and transfusion requirements in patients with paroxysmal nocturnal hemoglobinuria. N Engl J Med 2004;350:552-559.

16 Hillmen P, Young NS, Schubert J, Brodsky RA, Socie G, Muus P, Roth A, Szer J, Elebute MO, Nakamura R, Browne P, Risitano AM, Hill A, Schrezenmeier H, Fu CL, Maciejewski J, Rollins SA, Mojcik CF, Rother RP, Luzzatto L: The complement inhibitor eculizumab in paroxysmal nocturnal hemoglobinuria. N Engl J Med 2006;355:1233-1243.

17 Holers VM: The spectrum of complement alternative pathway-mediated diseases. Immunol Rev 2008;223:300-316

18 Weitz M, Amon 0, Bassler D, Koenigsrainer A, Nadalin S: Prophylactic eculizumab prior to kidney transplantation for atypical hemolytic uremic syndrome. Pediatr Nephrol 2011;26:1325-1329.

-19 Nester C, Stewart Z, Myers D, Jetton J, Nair R, Reed A, Thomas C, Smith R, Brophy P: Pre-emptive eculizumab and plasmapheresis for renal transplant in atypical hemolytic uremic syndrome. Clin J Am Soc Nephrol 2011;6:1488-1494.

-20 Zimmerhackl LB, Hofer J, Cortina G, Mark W, Wurzner R, Jungraithmayr TC, Khursigara G, Kliche KO, Radauer W: Prophylactic eculizumab after renal transplantation in atypical hemolytic-uremic syndrome. N Engl J Med 2010;362:1746-1748.

21 Davin JC, Gracchi V, Bouts A, Groothoff J, Strain L, Goodship T: Maintenance of kidney function following treatment with eculizumab and discontinuation of plasma exchange after a third kidney transplant for atypical hemolytic uremic syndrome associated with a CFH mutation. Am J Kidney Dis 2010;55:708-711.

22 Chatelet V, Fremeaux-Bacchi V, Lobbedez T, Ficheux M, Hurault de LB: Safety and long-term efficacy of eculizumab in a renal transplant patient with recurrent atypical hemolytic-uremic syndrome. Am J Transplant 2009;9:2644-2645. 